Proceedings

\title{
Exploring the Philosophical Problems of Artificial Intelligence Based on ERP Experiment ${ }^{+}$
}

\author{
Yuan Yan ${ }^{1,2}$ \\ 1 School of Humanities and Social Sciences, Xi'an Jiaotong University, Xi'an 710049, China; \\ yuanyu1007@126.com \\ 2 School of Business, Xi'an FanYi University, Xi'an 710105, China \\ + Fourth International Conference on Philosophy of Information, Berkeley, CA, USA, 2-6 June 2019.
}

Published: 4 June 2020

\begin{abstract}
To study the cognitive process of the human brain in dealing with philosophical issues, for the first time, from the perspective of scientific experiments, the issue of "relationship" in philosophy was verified. A set of algorithms combining physiology analysis and computer technology linearity and a nonlinear manifold learning algorithm were proposed. Two groups of auditory cognitive experiments were performed, and the concept expected effect was defined as the symbol of conceptual intervention. From the perspective of time, whether the concept was involved after the sensation arises was explored. EEG (electroencephalogram) physiology was used to analyze the data. The results showed that the concept induced a positive shift of the waveform after intervention. It has little effect on the early components, but it has a significant effect on the composition of the sensory components. Waveform changes before and after conceptual intervention have significant main effects. Perceptual production does not involve conceptual intervention, which verifies, in time, that "the relationship" exists.
\end{abstract}

Keywords: philosophical problems; event-related potential; manifold learning; ERP experiment

\section{Introduction}

Knowledge is divided into two kinds in the definition of philosophy: prior knowledge and experience knowledge. Prior knowledge refers to knowledge that naturally exists and does not depend on objective experience. Experience knowledge refers to the knowledge that people can feel their existence. In short, it is through feeling to feel everything in the world [1] The dispute between empiricism and transcendentalism has been long overdue. It can be traced back to the era of Plato. Plato believes that knowledge and feeling are two different things. The philosopher Protagoras believes that man is the yardstick of everything in the world. People's feelings about things come from the appearance of things to people [2]. After the rise of modern physics, the philosophical community further developed discussions on methodological issues. Aristotle sent a distinction between analytical methods and comprehensive methods. Galileo's analysis methods and synthesis methods are used to explain and propose hypotheses. Newton distinguishes from the perspective of cause and effect, and thus shifts to the direction of empiricism [3]. Since then, empirical methods include analysis. In Locke's time, he put forward a harsh criticism on the innate idea, which caused a far-reaching impact. At the same time, with the development of scientific practice, empirical theory has achieved great success. Many modern philosophers oppose Plato's a priori theory. This also makes the antithetical empiricism more and more important in the history of western philosophy [4].

Cognitive neuroscience is developed at the cross level of cognitive psychology, philosophy, artificial intelligence, artificial neural network, biology, physics, mathematics and other disciplines. It aims to elucidate the neurological mechanisms of mental history and how the operation of the brain 
produces psychological or cognitive functions, that is, to study how the brain creates spirit $[5,6]$. Common methods include psychophysics, electrophysiology, behavioral genetics, functional neuroimaging, and cognitive genetics. The main purpose is to explore how humans use neural molecules, brain cells, brain tissue regions, and whole brains to realize the neural mechanisms of human advanced mental activities, such as consciousness, language, memory, feeling, thinking, association, and imagination. At present, the research topics mainly include attention, consciousness, decision-making, learning, memory, etc. [7].

\section{Experimental Part}

Humans have the same or a similar perception of the objective world. Therefore, it is feasible to give the subject a certain concept description through experiments. From the Internet and CD, 120 kinds of sounds in daily life were selected. Adobe Audition CS6 software was used to intercept 1000 ms to maintain its identifiability. Then, the sound quality lossless normalization process is performed. The 30 participants who participated in the pre-experiment were auditioned, one by one, to record the concept of sound. Based on familiarity with the sound, scores were divided into five levels (5 points: very familiar, four points: relatively familiar, three points: somewhat familiar, two points: unfamiliar, one point: completely unfamiliar). The 80 voices with the highest identification (score) were counted for the main experiment, including 12 animal calls.

The experiment includes two conditions: The experimental group voice prompts: each sound is in a group of "blurry mixing, fuzzy mixing, voice prompts, fuzzy mixing" order, and 80 kinds of sounds are presented randomly. Before each group of sounds is presented, a black " + " shape is presented in the center of the light grey screen for $500 \mathrm{~ms}$, followed by $1000 \mathrm{~ms}$ of sound stimulation. Each segment of sound is randomly spaced at intervals of 1500 to $2000 \mathrm{~ms}$. The experiment was divided into 80 groups. After the presentation of the 16th, 32nd, 48th, and 60th groups of voices, the participants can take a short break. The control group text prompts: each sound is randomly and sequentially presented in the order of "fuzzy mixing, fuzzy mixing, text prompting, and fuzzy mixing". In the following, the entirety of the first time presented fuzzy sound under both conditions is simply referred to as " 1 blur". By analogy, they are called " 2 blurs", " 3 prompts", and " 4 blurs".

\section{Results and Discussion}

\subsection{Behavioral Results}

The main experiment of 19 groups and the control experiment of 9 groups were performed. The behavioral results of auditory cognition experiments were recorded under the conditions of the two sensory channels.

The reaction time results showed that the average response time of the experimental group was 21.92 ms lower than that of the control group. There was no significant difference between groups. The correct rate results showed that the correct rate of the experimental group was slightly higher than that of the control group by 0.98 percentage points. There was no significant difference between groups.

It was found that the average response time of the same sensory channel (auditory) was 21.92 $\mathrm{ms}$ faster than that of the control group. Error rates under voice and text prompts were $5.88 \%$ and $6.86 \%$, respectively, and both were less than $10 \%$. Therefore, it is in line with the task requirements of the subjects. The specific results are shown in Table 1 . There was no difference between groups in the two groups. The miss ratio in the experimental group was nine percentage points smaller than that in the control group. After the prompt, the hit rate of the subjects who chose the new different stimulus was significantly higher than when they were not prompted $(F(1,27)=59.67, p<0.001)$. 
Table 1. Behavioral results and levels of difference.

\begin{tabular}{ccccc}
\hline Condition & Reaction Time (ms) & Error Rate (\%) & Miss Ratio (\%) & Hit Rate after Prompt (\%) \\
\hline Voice prompts & $1135.40(281.64)$ & $5.88(2.72)$ & $25.55(14.65)$ & $97.02(0.82)$ \\
Text prompts & $1157.32(263.03)$ & $6.86(2.68)$ & $34.26(13.86)$ & $94.44(1.05)$ \\
\hline
\end{tabular}

\subsection{ERP Results}

In order to eliminate the effects of new and different stimuli and differences in physical characteristics, two blur (pre-prompt) and four blur (post-prompt) waveforms were analyzed. According to the two blur (pre-prompt) and four blur (post-prompt) electrodes of the experimental group and the control group at the electrodes of F3, FZ, F4, C3, CZ, C4, P3, PZ, and P4, the fuzzy evoked a relatively uniform waveform. This is similar to P1, N1, P2, N2 and other components. On the whole, the conceptual expectation effect (conceptual intervention) induces a positive shift in the ERP waveform. The difference wave between 4 blur and 2 blur is a positive wave with a large span.

0 200 ms after stimulation: The experimental and control data for the P1, N1 components of the brain regions corresponding to this time window were compared with two blurs (pre-prompt) and four blurs (post-prompt). The waveform has a high degree of overlap in the forehead area and the center area and is slightly separated in the top area. Among them, the peak value of the N1 component of the experimental group data was corrected in the right hemisphere, and the control group was more negative. The results of ANOVA repeated measurement analysis are shown in Tables 2 and 3. Two blur/four blur has no significant main effect. Electrode locations (between the anterior and middle regions of the brain) were not significantly different between the hemispheres of the brain (between the left, middle and right regions of the brain). There is no interaction between two blur/four blur and the brain's electrode position and hemisphere. There is a significant interaction between electrode location and hemisphere location. Reaction type, hemispherical site, and electrode position have no obvious interaction effect. The data in the control group were not significant. This shows that there is a small difference in early auditory processing before and after the voice prompts and text prompts.

Table 2. The results of $0 \sim 200 \mathrm{~ms}$ in the experimental group.

\begin{tabular}{cccc}
\hline Effect & F & P & Partial Eta Square \\
\hline Two blur/four blur & 2.021 & 0.181 & 0.144 \\
Brain area distribution & 3.005 & 0.091 & 0.353 \\
Brain lateralization & 7.804 & 0.008 & 0.587 \\
Two blur/four blur *brain area distribution & 0.620 & 0.556 & 0.101 \\
Two blur/four blur *brain lateralization & 0.718 & 0.509 & 0.115 \\
Brain area distribution *brain lateralization & 7.338 & 0.007 & 0.765 \\
\hline
\end{tabular}

Table 3. The results of $0 \sim 200 \mathrm{~ms}$ in the control group.

\begin{tabular}{cccc}
\hline Effect & F & P & Partial Eta Square \\
\hline Two blur/four blur & 0.000 & 0.993 & 0.000 \\
Brain area distribution & 5.196 & 0.060 & 0.675 \\
Brain lateralization & 0.036 & 0.965 & 0.014 \\
Two blur/four blur *brain area distribution & 2.176 & 0.209 & 0.465 \\
Two blur/four blur *brain lateralization & 3.147 & 0.130 & 0.557 \\
Brain area distribution *brain lateralization & 1.120 & 0.482 & 0.599 \\
Two blur/four blur *brain area distribution *brain lateralization & 3.503 & 0.166 & 0.824 \\
\hline
\end{tabular}

200 400 ms after stimulation: The experimental group data and the control group data were compared in the P2, N2 components of the brain area of the time window, two blurs (pre-prompt) and four blurs (post-prompt). There is a more correct offset for the waveform after the prompt. Differences in experimental data were more significant than those in the control group. The results 
of ANOVA repeated measurement analysis are shown in Tables 4 and 5. The two blur/four blur of the experimental group has a significant main effect. There was no significant difference between the anterior and middle apical regions of the brain in the electrode position. The hemispherical location of the brain (between the left, middle and right regions of the brain) is significantly different two blur/four blur and the brain's electrode position and hemisphere have no interaction effect. There is no significant interaction between the electrode position and the hemisphere. In the control data, only two blur and four blur have significant main effects.

Table 4. The results of $200 \sim 400 \mathrm{~ms}$ in the experimental group.

\begin{tabular}{cccc}
\hline Effect & F & P & Partial Eta Square \\
\hline Two blur/four blur & 6.714 & 0.024 & 0.359 \\
Brain area distribution & 1.388 & 0.290 & 0.201 \\
Brain lateralization & 3.991 & 0.050 & 0.421 \\
Two blur/four blur *brain area distribution & 0.480 & 0.631 & 0.080 \\
Two blur/four blur *brain lateralization & 1.125 & 0.359 & 0.170 \\
Brain area distribution *brain lateralization & 2.161 & 0.155 & 0.480 \\
Two blur/four blur *brain area distribution *brain lateralization & 1.287 & 0.345 & 0.364 \\
\hline
\end{tabular}

Table 5. The results of 200 400 $\mathrm{ms}$ in the control group.

\begin{tabular}{cccc}
\hline Effect & F & P & Partial Eta Square \\
\hline Two blur/four blur & 3.233 & 0.050 & 0.144 \\
Brain area distribution & 2.842 & 0.150 & 0.353 \\
Brain lateralization & 1.723 & 0.270 & 0.587 \\
Two blur/four blur *brain area distribution & 1.163 & 0.385 & 0.101 \\
Two blur/four blur *brain lateralization & 0.770 & 0.511 & 0.115 \\
Brain area distribution *brain lateralization & 5.046 & 0.099 & 0.765 \\
Two blur/four blur *brain area distribution *brain lateralization & 3.122 & 0.188 & 0.320 \\
\hline
\end{tabular}

\section{Conclusions}

The problem of philosophical knowledge is combined with the cognitive neuron-brain experiment. Through two sets of ERP experiments, the participants were tested for sound recognition in the experimental group "fuzzy mixing, fuzzy mixing, voice prompts, fuzzy mixing "and the control group "fuzzy mixing, fuzzy mixing, text prompting, fuzzy mixing". The following conclusions are drawn. From the results of behavioral studies, subjects in the experimental group were all the same sensory channels. The reaction time is faster. The hit rate of the second choice was significantly higher than that of the unprompted group. The subjects changed from auditory perception to conceptual change. From the data of electroencephalogram, sound materials induce early auditory stimulus processing components. The waveform is close to the same in the $0 \sim 200 \mathrm{~ms}$ time period. There are significant differences in the 200 400 ms time period. It reflects the brain's advanced cognitive processing of sound stimulation. Therefore, it has been verified temporally that the concept involves later than sensory occurrence. It verifies from time that "the relationship" exists.

\section{References}

1. Hutson, M. AI Glossary: Artificial intelligence, in so many words. Science 2017, 357, 19.

2. Byrne, M.F.; Chapados, N.; Soudan, F.; Oertel, C.; Pérez, M.L.L.; Kelly, R. Su1614 artificial intelligence (AI) in endoscopy-Deep learning for optical biopsy of colorectal polyps in real-time on unaltered endoscopic videos. Gastrointest. Endosc. 2017, 85, AB364-AB365.

3. Etzioni, O. Artificial intelligence: AI zooms in on highly influential citations. Nature 2017, 547, 32.

4. Altman, R.B. Artificial intelligence (AI) systems for interpreting complex medical data sets. Clin. Pharmacol. Ther. 2017, 101, 585-586. 
5. Hassabis, D.; Kumaran, D.; Summerfield, C.; Botvinick, M. Neuroscience-inspired artificial intelligence. Neuron 2017, 95, 245-258.

6. Wang, M.; Zhao, L.; Du, R.; Wang, C.; Chen, L.; Tian, L. A novel hybrid method of forecasting crude oil prices using complex network science and artificial intelligence algorithms. Appl. Energy 2018, 220, 480-495.

7. Li, D. Artificial intelligence in the rising wave of deep learning: The historical path and future outlook. IEEE Signal Process. Mag. 2018, 35, 180-177.

(C) 2020 by the authors. Licensee MDPI, Basel, Switzerland. This article is an open access article distributed under the terms and conditions of the Creative Commons Attribution (CC BY) license (http://creativecommons.org/licenses/by/4.0/). 